\title{
THEORETICAL CALCULATIONS \\ OF INTERSTELLAR CLOUD FORMATION
}

\author{
DONALD W. GOLDSMITH* \\ State University of New York, Stony Brook, N.Y., U.S.A.
}

\begin{abstract}
Numerical calculations of the growth of thermal instabilities show that condensations tend to form with the proper sort of density contrast but the wrong shapes (and, to some extent, sizes) to qualify as the observed interstellar clouds analyzed by Heiles.
\end{abstract}

During the last few years, several theoretical models for the heating and ionization of the interstellar medium have been proposed (cf. Grewing (1974)). Each of these models for the overall behavior of the medium allows for a calculation (by numerical hydrodynamics) of how small perturbations in the local temperature and density might grow into sizable contrasts in these parameters that could pass for observed interstellar 'clouds'. Such calculations have been carried out in some detail by Goldsmith (1970) for the steady-state model based on heating by low-energy cosmic rays or X-rays, by Schwarz et al. (1972) for the time-dependent model of heating by localized supernova outbursts, and by Ames (1973) for the steady-state model with the inclusion of the Rayleigh-Taylor instabilities proposed by Parker (1966) that are important for perturbation wavelengths of $\sim 1 \mathrm{kpc}$ or more.** All of these calculations (cf. the review by Goldsmith, 1973) show that if the assumed parameters of the model do indeed characterize the interstellar medium, then condensations with a density increase of $\sim 10^{2}$ over the original density are likely to form. This is, of course, good news from the theoretical front.

The bad news is that the condensations which are formed by the numerical hydrodynamics tend to have dimensions less than those of 'typical' interstellar clouds, and, worse, that the condensation process occurs along the magnetic lines of force, producing flat pancakes for clouds. Unfortunately, as Heiles (1974) has shown in his review of interstellar $\mathrm{H} \mathrm{I}$ in this volume, cloud material appears in filaments elongated along the magnetic field lines (as estimated from optical polarization measurements). Before we therefore abandon the numerical calculations, let us recall that they are the only calculations we have, and look briefly at the fundamental mechanism of thermal instability.

As outlined in Field's classic paper (1965), the process of thermal instability hinges on the possibility that gas somewhat cooler than its surroundings may quickly become much cooler. Because of the perfect gas law, such gas will most likely become much denser as it cools by radiating optical and infrared photons. For the interstellar medium, the approximation that the gas is optically thin to its own radiation ranks

\footnotetext{
* Present address: 1655 12th Ave., San Francisco, Calif. 94122.

** Because of the complexity of the problem studied by Ames, her calculations concern only the linearized hydrodynamical equations and do not enter the non-linear region of sizable density contrasts.
} 
among the safest of the many that enter the calculations. In the steady-state model

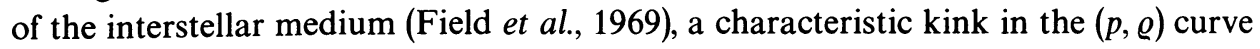
for thermal and ionization equilibrium that occurs for temperatures near $6000 \mathrm{~K}$ leads naturally to a situation where a slight density increase (or temperature decrease) produces a phase transition from hot, rarefied gas $\left(T \simeq 6000 \mathrm{~K}, n \simeq 0.2 \mathrm{~cm}^{-3}\right)$ to a cold, dense gas $\left(T \simeq 20 \mathrm{~K}, n \simeq 40 \mathrm{~cm}^{-3}\right)$ in a time of $2-3 \times 10^{7} \mathrm{yr}$. On the other hand, the calculations by Schwarz et al. (1972) of thermal instability within a medium that is cooling and recombining after a supernova outburst show that a similar density increase will arise from an initially small $(10 \%)$ perturbation, but in a time of $10^{6} \mathrm{yr}$.

The maximum wavelength affected by the thermal instability is in both cases approximately $c t$, where $c$ is the speed of sound in the initial medium, about $8 \mathrm{~km} \mathrm{~s}^{-1}$, and $t$ is the time mentioned above. Thus the steady-state and time-dependent models have maximum wavelengths for the initial perturbation of $\sim 10^{20.5}$ or $10^{19} \mathrm{~cm}$ respectively. These perturbations could yield spherical clouds with acceptable sizes of $10^{20}$ or $10^{18.5} \mathrm{~cm}$ for the largest clouds formed, if the condensation could proceed in three dimensions. However, if the instability occurs in regions with initial magnetic fields of $1 \mu \mathrm{G}$ or more, then the contraction perpendicular to the field lines cannot keep up with the contraction along the field lines after the first $e$-folding of the density. The result would be a cloud with a thickness of $\sim 10^{18.5}$ or $10^{17} \mathrm{~cm}$ along the field lines, but a hundred or more times wider in directions perpendicular to the field. Although accurate data on the sizes and shapes of clouds are lacking, such observations as are now available (Heiles, 1974) fail to reproduce the calculated models. The discrepancy between the elongation predicted for theoretical clouds and the actual (reverse) elongation that has been observed powerfully reminds us that we have no reliable understanding of how clouds form in the presence of a magnetic field. A realistic (more than one-dimensional) treatment of the numerical magnetohydrodynamics of condensations may someday provide the key to resolving this problem.

Meanwhile, in 1973, two conclusions emerge from the theoretical studies. First, condensations will form through thermal instabilities in a gas that is cooling from $T \simeq 10^{4} \mathrm{~K}$ and recombining after a supernova outburst. The size, and hence the overall importance, of the regions subject to such condensation processes depends on the flux of ionizing photons from the supernova. A small ionizing flux $\left(\ll 10^{51} \mathrm{erg}\right.$ ) would make the Schwarz et al. (1972) condensations a relatively local accompaniment of supernova outbursts. Second, if the interstellar medium is heated and ionized primarily by a steady flux (steady on time scales greater than $10^{7} \mathrm{yr}$ ) of low-energy cosmic rays or X-rays, then thermal instabilities will produce condensations wherever the local density exceeds a certain critical value (near $n=0.2 \mathrm{~cm}^{-3}$ with the assumed model parameters). The significance of this mechanism depends on the importance of the steady flux as compared to the time-dependent sources of heating and ionization. If the ionization rate from the unmeasured portions of the X-ray and cosmic-ray spectra does not exceed that from the measured X-ray background, then steady-state 
theories can not explain the observed temperatures in interstellar clouds, and are unlikely to explain the formation of these clouds.

Calculations of the thermal instabilities that could produce interstellar clouds suffer from gross uncertainties concerning the initial conditions that could lead to condensations. Such uncertainties can be invoked in partial explanation of why clouds formed in computers differ from clouds we find in our Galaxy, but apparently some key element of the problem still eludes us. Cox (1973) has suggested that supernova blast waves play the dominant role in ordering the interstellar medium, and perhaps this favorite theoreticians' retreat, the supernova event, can thus save us once again.

\section{References}

Ames, S.: 1973, Astrophys. J. 182, 387.

Cox, D.: 1973, paper presented at the meeting of IAU Commission 34, Sydney, Australia.

Field, G.: 1965, Astrophys. J. 142, 531.

Field, G., Goldsmith, D., and Habing, H.: 1969, Astrophys. J. Letters 155, L149.

Goldsmith, D.: 1970, Astrophys. J. 161, 41.

Goldsmith, D.: 1973, review paper presented at the meeting of IAU Commission 34, Sydney, Australia

(available as report no. 560 of CRSR, Cornell University).

Grewing, M.: 1974, this volume, p. 97.

Heiles, C.: 1974, this volume, p. 13.

Parker, E.: 1966, Astrophys. J. 145, 811.

Schwarz, J., McCray, R., and Stein, R.: 1972, Astrophys. J. 175, 673.

D. W. Goldsmith

1655 12th Ave.,

San Francisco, Calif. 94122, U.S.A.

\section{DISCUSSION}

Habing: It is possible to obtain $n_{\mathrm{e}}$ in any specified cloud from interstellar absorption lines of an element in two ionization stages. Dunham tried this already in 1939. This probably requires higher spectral resolution than is available even with the Copernicus satellite.

Goldsmith: This is probably the best way to determine the electron density accurately, and in the few cases where $\mathrm{Ca}$ II/CaI line strengths have been measured (by White), it has already given valuable results. Also, the MgII doublet can be used in a similar way if we can observe at the appropriate near-ultraviolet wavelength.

Jenkins: I'd like to ask Grewing about how dense a cloud in your calculations of the ionization equilibrium complies with this. You must have a problem when it gets so dense that it is opaque to X-rays in the cloud bed. Is that in a regime of observability with optical ultraviolet lines?

Greenberg: And I would like to ask, in your calculation of the reduced cooling rate due to the evidence of reduced abundance of the heavier elements based on Copernicus, did you then take into account the additional cooling due to the additional dust and consequent molecules?

Grewing: Our model calculations were not really aimed at treating interstellar clouds but rather the intercloud gas. To treat clouds adequately, we would have to incorporate further heating and cooling processes into our computer program. We are in the process of doing this.

Goldsmith: The cloud and intercloud medium could be heated in entirely different ways. Soft X-rays may heat the intercloud medium, but almost certainly they don't penetrate the clouds. You need another explanation. It may be some unknown kind. Then again it would not be surprising that we don't know much about how to form clouds.

Gordon: As Burke mentioned, perhaps the association of magnetic fields with filaments means only that 
the matter controls the fields and not the other way around. In this case, we should ignore the fields in modeling the formation of clouds.

Goldsmith: We still seem to face the problem either of moving material across field lines on time scales $\sim 10^{7} \mathrm{yr}$ (or less), or of explaining the compression of material into clouds without raising the field strength by two orders of magnitude.

Oort: How about a supernova explosion? You can form clouds in a supernova explosion.

Goldsmith: Perhaps everything else is ruled out and you do need a huge energy input. You still need an extra power of linkage because otherwise the magnetic field will dominate.

Kahn: Do you not think that one ought to compare the magnetic energy density with the turbulent energy density in interstellar space, in order to decide whether the cloud motions can distort the magnetic field pattern?

Goldsmith: It seems that the greatest problem is not to have clouds distort the magnetic field pattern, but rather to explain how an initial perturbation in the intercloud medium can become a cloud elongated along the magnetic field.

Townes: In the case of dense molecular clouds, many shape irregularities have been found, but none so far can be described as filamentary. In this case also, the kinetic energy density is probably much greater than the magnetic field energy density, so that one has a simpler, or at least a different, theoretical problem.

While observations of dense clouds characteristically indicate blob-like shapes, with great irregularities rather than filaments, it is also clear that in some cases there is finer structure within the clouds that is not yet resolved. This finer structure might possibly be filamentary, but there is no such indication at present.

Goldsmith: Dense (molecular) clouds may indeed not suffer from the same problems of magnetic field restrictions as those which arise in the formation of 'ordinary' clouds. Unfortunately we probably still must form 'ordinary' clouds on the way to forming dense clouds.

Davies: In reply to Goldsmith's query whether the magnetic field penetrates $\mathrm{HI}$ clouds, I would point out that Zeeman measurements show that the denser $\left(n_{\mathrm{H}} \simeq 100 \mathrm{~cm}^{-3}\right)$ clouds are permeated by magnetic fields. Further, the $\mathrm{OH}$ maser sources $\left(n_{\mathrm{H}} \sim 10^{6}\right.$ to $\left.10^{7} \mathrm{~cm}^{-3}\right)$ contain fields whose magnitude is that expected from the compression of these dense Hi clouds on the assumption of flux conservation. Magnetic fields of the observed strengths play a significant role in the pressure equilibrium of all these clouds.

Goldsmith: However, the key question that remains is how to form clouds from intercloud material without being prevented (by the magnetic field) from contracting in directions perpendicular to the field.

Burke: The Crab nebula is the best-observed example of a magnetic, hot gas which exhibits condensations. Have you considered this example?

Goldsmith: The formation of condensations in material ionized by a supernova explosion has been considered by Schwarz, McCray and Stein, and by Kafatos and Morrison. I think it more difficult to form interstellar clouds in this way than to form such localized filaments. 